\title{
CEPHALOTUS FOLLICULARIS CULTIVARS AND FORMS IN CULTIVATION - IS THERE A BASIS FOR THE CURRENT NAMING PROTOCOLS?
}

\section{RICHARD NUNN・richardjnunn1@gmail.com}

Keywords: Cephalotus follicularis, cultivation, cultivar.

Over the past few years the author has watched with interest as cultivators of Cephalotus follicularis have attempted to find some points of difference to apply cultivar names, some registered, most not. Unfortunately, in what appears to be a push for commercial gain, the current plethora of cultivar names has reached the point of ridiculous, and one which unfortunately is also gaining popularity with other genera of carnivorous plants. Human nature is such that when we get bitten by the collecting bug we want to have every species or variation of it in our collections and I fear that this is now occurring with $C$. follicularis. The objective of this article is to try and bring some common sense to the naming of $C$. follicularis forms and preventing collectors from wasting their money on dubious and spurious forms and cultivars of this plant. Having, over the past 30 years cultivated this genus (with varying levels of success) and in the past decade documented and photographed this plant in multitudes of locations in its natural habitat, I feel that I have some basis for passing comment on this topic. The assertions in this article are my own views, but are supported by similar views of authorities such as Phill Mann, Allen Lowrie, and Greg Bourke, who have seen and studied C. follicularis at multiple locations in its natural habitat and cultivated many clones of this plant.

For a number of years Phill Mann and I have been trying to find stable variations in C. follicularis, but in cultivation they always tend to revert to the mean, and plants from different sites that looked different in the field (see figures) tend to all end up looking pretty similar in the same conditions. Sure some plants color up a bit more and others might produce a larger pitcher, but it is often not the most colorful or largest pitchers from the field that translate into these specimens in cultivation. I think it is worth noting that any collected specimens were done legally under permit and only cuttings taken so as not to remove any plants. A simple comparison between $C$. follicularis and Dionaea and Sarracenia cultivars shows that the latter hold true to form when basic conditions such as light, temperature, and water levels are met, i.e. a Sarracenia 'Adrian Slack' tends to look the same for most growers who have a basic idea of how to grow them, the same cannot be said for C. follicularis. Sure if you want to grow them under high intensity

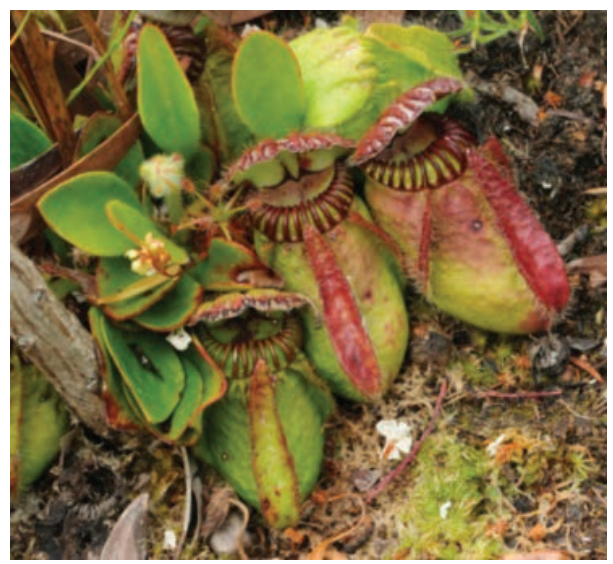

Figure 1: Cephalotus follicularis photographed at Torndirrup National Park. This plant produces particularly robust and large pitchers in the field. Perhaps it is the parent of Cephalotus 'Hummer's Giant' or is it just a plant that is growing in favorable conditions? 

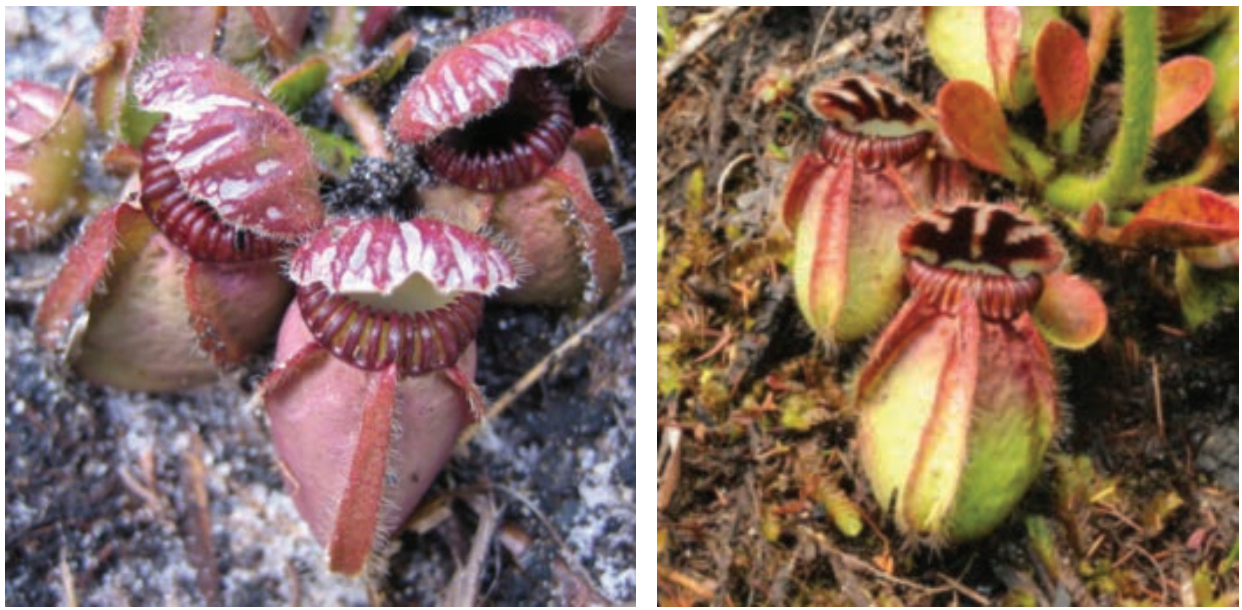

Figure 2: Cephalotus follicularis photographed at Broke Inlet (left) and at Donnelly River (right).
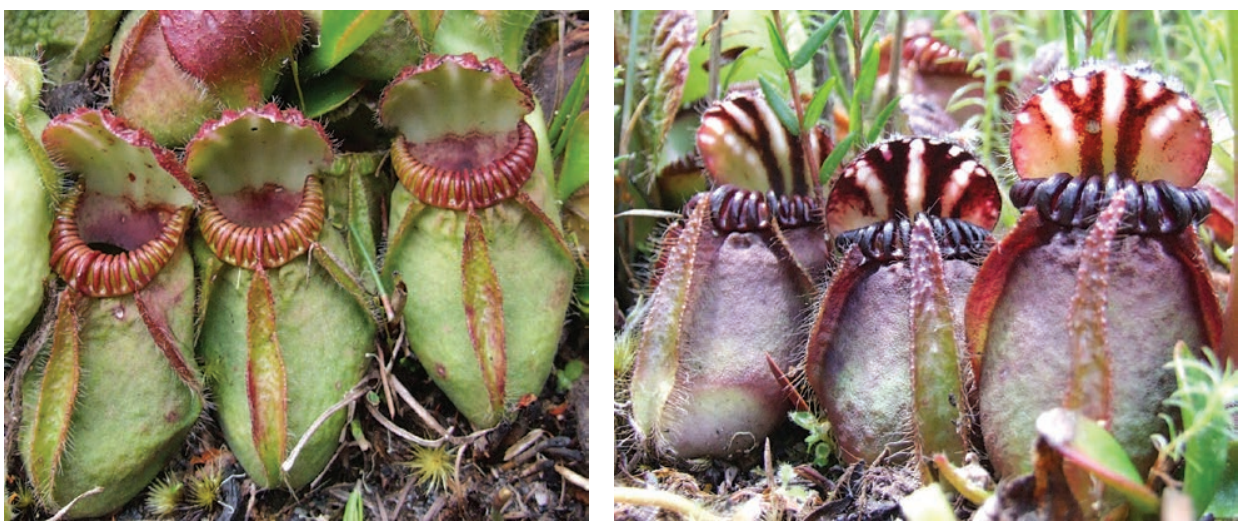

Figure 3: Two plants of Cephalotus follicularis showing different coloration photographed on the Denmark - Mt. Barker Road.

light they will color up and some will show different shades to others, but this is not how they grow in the field.

Having seen many natural sites and cultivated plants over the years, the conclusion I arrive at is that $C$. follicularis is very reactive to its micro habitat. There are some broad generalizations that hold true, in bright light the plants will color up and the pitchers tend to be smaller and more robust, in shade the pitchers can attain impressive dimensions and tend to stay green or lightly colored. However, to add to the confusion, plants in the field have been observed that behave in the opposite manner. In fact, at most sites in the wild, C. follicularis can be quite variable in terms of color, pitcher size, and growth habit. Often two plants growing next to each other will have different color and size. So what causes this variation, is it genetic or is it the micro habitat variables, such as nutrients, water, light, substrate, and temperature variation, all of which can vary subtly across one individual location? To be clear, the scientific research hasn't been carried out to answer this conclusively. However, my observations, and those of others that have significant knowledge of this species 

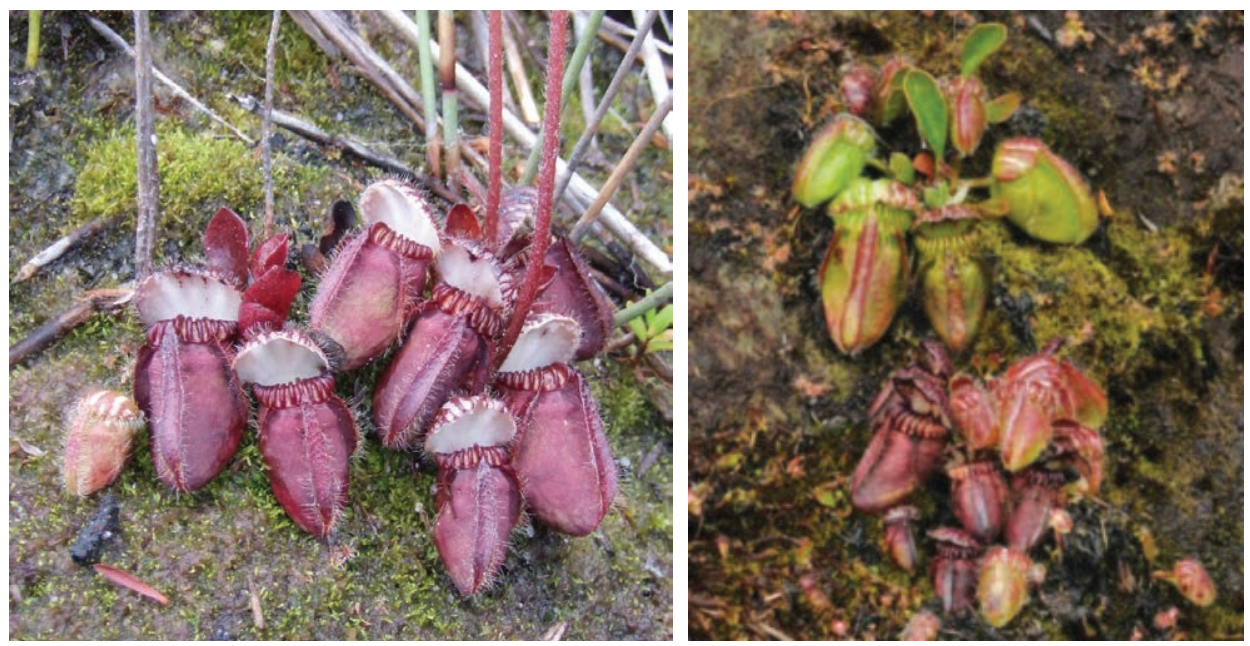

Figure 4: Plants showing deep red color due to constant exposure to sunlight at Coalmine Beach. The image on the right has a plant growing in the same light, but hasn't taken on the same level of coloring, which is likely due to subtle differences in nutrients rather than genetic variation.
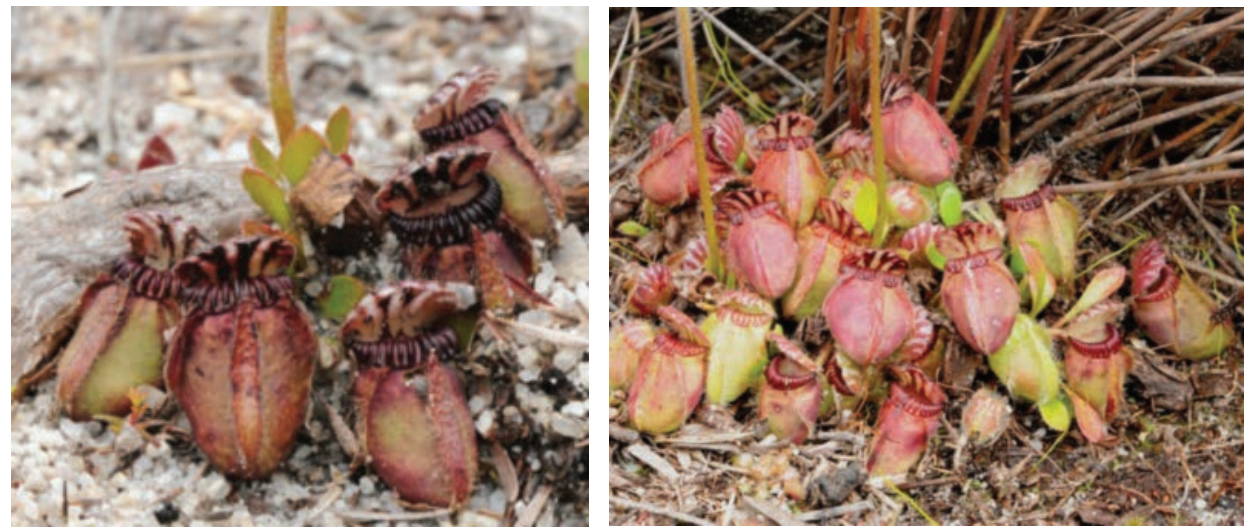

Figure 5: Cephalotus follicularis growing in unusual conditions at Northcliffe in pure sand, which reflects the sunlight. Consequently, the plants are highly colored and the pitchers are smaller due to the harsh conditions.

both in its natural habitat and in cultivation, would point towards differences being environmentally induced rather than genetically.

Currently there are three registered C. follicularis cultivars, 'Eden Black', 'Hummer's Giant', and 'Clayton's T Rex', all of which I would question the validity. In no way do I want to down play the work done by the authors of these cultivars. I think we would all like to believe there are different forms of $C$. follicularis worthy of our collections; however, I have always been skeptical, because fundamentally $C$. follicularis differences are unstable in cultivation. They may hold true for one grower with unique conditions and for the next with good, but slightly different growing conditions, they won't color up or the pitchers will only be of an average size. 

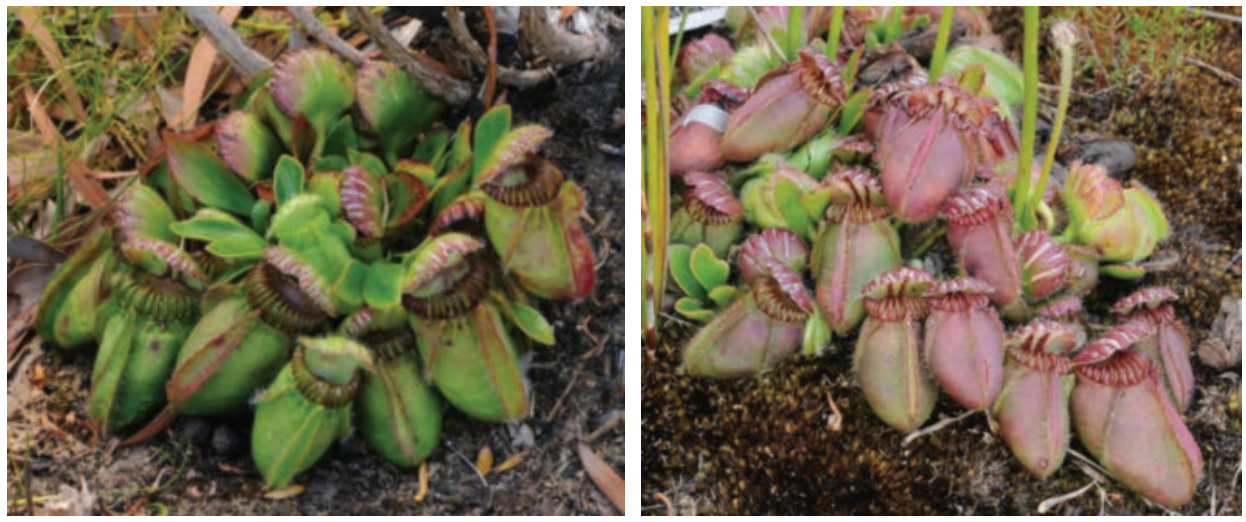

Figure 6: Two large plants of Cephalotus growing in the Torndirrup National Park. These images highlight the variability of Cephalotus at the same site in the same light conditions. Tests in cultivation suggest that these color and pitcher size traits are not stable and are extremely reliant on the microhabitat around the plant.
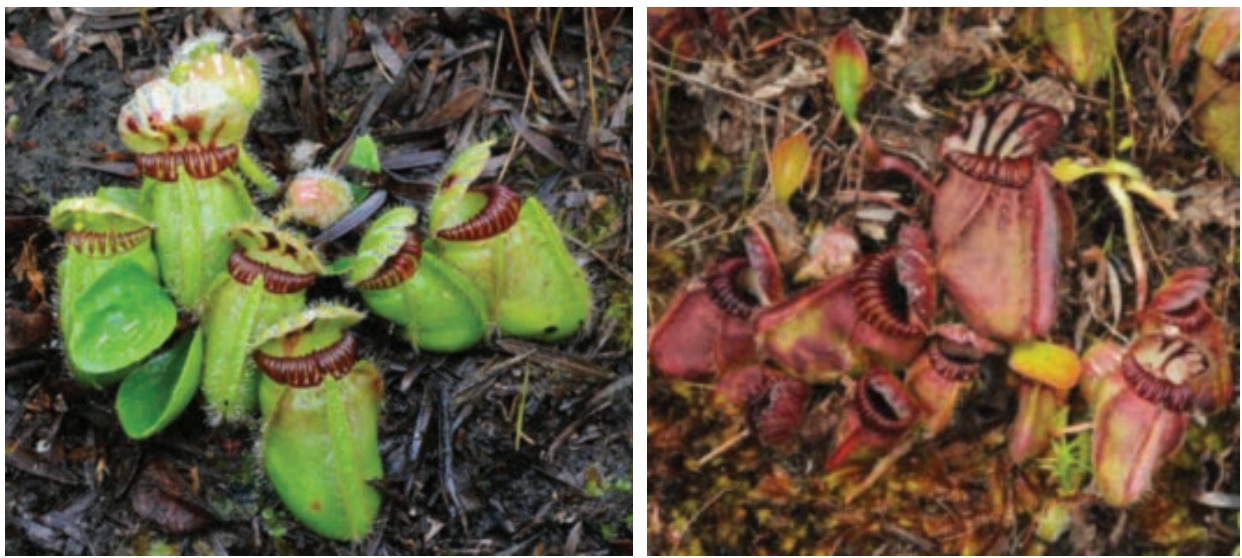

Figure 7: A plant photographed at Mount Frankland National Park (left) and a deeply colored plant growing in the Two Peoples Bay area (right).

Along the same lines is the disturbing emergence of names such as "Big Boy", "German Giant", "vigorous clumping", and "squat". These names are just simply not valid and there is enough evidence on the web to suggest that growers are struggling to find much difference in these clones in their conditions. The bottom line is they aren't stable and people are wasting their money.

Where things have got really stupid is the emergence of names such as "Phill Mann" or "Allen Lowrie" being put on these plants and people actually collecting them. I can understand collectors putting the source of their plants on the back of the label, but to actually use this as a point of difference is ridiculous. In fact, most of the original clones of $C$. follicularis in cultivation today originated from Allen or Phill some 30 plus years ago, others would have been from plants collected by botanic gardens. Either way, the provenance of these plants is patchy to say the least. Let's use $C$. follicularis "Phill Mann" as an example. Over many years Phill has built up stock from legally collected cuttings (that means under permit) from plants from many sites. I have been in his greenhouse and they are all mixed up. Same story with Allen Lowrie, (again from legally collected cuttings) 
who clearly markets his plants as from a mix of multiple clones and sites. So if you are buying a piece of plant that has been labeled "Phill Mann" or "Allen Lowrie", which clone are you getting, what location is it from? No one really knows. This is hardly a sensible way to categorize cultivars of $C$. follicularis.

It is easy to sit here and criticize attempts to categorize or split out different forms of $C$. follicularis without offering an alternative. I therefore propose that there is a more sensible approach to this issue. We are now seeing a few plants with location names entering cultivation, plants such as "Coalmine Beach" and "Two People's Bay". The problem for collectors is that the collection of these plants from the wild is strictly regulated and only a very few people have the necessary permits, particularly as the majority of $C$. follicularis sites are in National Parks. It will require some patience for properly named location clones of $C$. follicularis to enter cultivation, but over time it will happen. Knowing the provenance of a plant is valuable for several reasons, not least being that if a site is destroyed by man-made activity or act of nature, then we have a back-up in cultivation of genetic material that would otherwise be extinct. Also we know that $C$. follicularis is not stable in cultivation and it would make for interesting comparisons if collectors could discuss the variations in their plants from the same location. This is the same naming protocol that has taken hold with other genera of CPs. Specifically, Sarracenia and Nepenthes have been classified in cultivation using location names for some time.

I have no doubt this article will raise some debate, but I hope that some sanity will prevail and that the current naming of $C$. follicularis forms, which cynically seems to be aimed at commercial gain, will not take hold. Also the scientists in the CP community will probably criticize the lack of true research and data to support the claims in this work, but perhaps also it might encourage more detailed field research to unlock the secrets of this enigmatic species.

\section{(9) petflytrap}

- Mail order and Internet U.S. sales

- Dionaea, Sarracenia, Nepenthes, Drosera, Pinguicula, books, supplies, and much more

-We stock multiple sizes of most plants

- Terrarium kits with live plants (no seeds)

- Rare nursery-grown, imported lowland/intermediate Nepenthes

- Most plants shipped potted and ready to grow!

\section{www.petflytrap.com \\ orders@petflytrap.com \\ 281-433-3286}

